\title{
Interstitial Pregnancy a Rare Form of Ectopic Pregnancy: Case Report
}

\author{
Mohannad Abu-FAZA', İbrahim A. ABDELAZIM1,2
}

Kuwait

\section{ABSTRACT}

Interstitial pregnancy is a rare variety of ectopic pregnancy, it can have disturbed 8-16 weeks of amenorrhea (later than the distal tubal ectopic pregnancy), due to the dispensability of the myometrium covering the interstitial segment of the fallopian tube, with subsequent massive internal hemorrhage.

A 29-years-old female, Gravida 3, Para 2, presented with severe abdominal pain, vomiting, and syncope 3 days after her missing period, and positive pregnancy test. Trans-vaginal ultrasound showed empty uterine cavity, free pelvic fluid with severe cervical motion tenderness. She was diagnosed with ruptured ectopic pregnancy. Ruptured ectopic pregnancy in the proximal segment of right fallopian tube was confirmed with laparotomy.

The ruptured pregnancy was managed by right salpingectomy, and hemostatic stitches at the right uterine corn. Her hemoglobin was $8 \mathrm{gr}$ on admission, and she received 3 units of packed RBCs (one intraoperative, and two post-operative). Her post-operative hemoglobin was $10.5 \mathrm{gr}$, and she was discharged from the hospital on the 3rd post-operative day in good general condition for follow up in the outpatients department.

This case report represents a rare variety of ectopic pregnancy, which is the interstitial pregnancy, because it can rupture few days or weeks after the missed period with subsequent massive internal hemorrhage.

Interstitial pregnancy is a rare form of ectopic pregnancy, the obstetricians should be aware of rare forms of ectopic pregnancies, it can have disturbed few days or weeks after the missed period leading to subsequent significant morbidity.

Keywords: Interstitial, Ectopic, Pregnancy, Rare

Gynecol Obstet Reprod Med 2018;24(3):167-169

\section{Introduction}

Interstitial pregnancy is a rare variety of ectopic pregnancy, occurs after implantation of the fertilized ovum in the proximal tubal segment that lies within the muscular uterine

${ }^{1}$ Department of Obstetrics and Gynecology Ahmadi Hospital Kuwait Oil Company (KOC), Kuwait

${ }^{2}$ Department of Obstetrics and Gynecology Ain Shams University Cairo, Egypt.

Address of Correspondence: Ibrahim A. Abdelazim

Professor of Obstetrics and Gynecology, Ain Shams University, Cairo, Egypt, and Ahmadi Kuwait Oil (KOC) Company

Hospital, Kuwait.

dribrahimanwar@gmail.com

Submitted for Publication: $\quad$ 19.10.2017

Accepted for Publication: $\quad$ 06. 01.2018

\begin{tabular}{|c|c|}
\hline & Access this article online \\
\hline $\begin{array}{c}\text { Quick Response Code: } \\
\text { 口. }\end{array}$ & $\begin{array}{c}\text { Website: www.gorm.com.tr } \\
\text { info@gorm.com.tr }\end{array}$ \\
\cline { 2 - 3 } & DOI:10.21613/GORM.2017.744 \\
\hline
\end{tabular}

How to cite this article: Faza MA. and Abdelazim IA. Interstitial Pregnancy a Rare form of Ectopic Pregnancy: Case Report. Gynecol Obstet Reprod Med 2017;24(3):167-169 wall (incorrectly called corneal pregnancy). Undiagnosed interstitial pregnancy usually disturbed at 8-16 weeks of amenorrhea (later than the distal tubal ectopic pregnancy). In addition; the interstitial ectopic pregnancy carries the risk of severe hemorrhage, and high mortality rate $(2.5 \%)$, due to the proximity of the interstitial fallopian tube to the uterine, and ovarian arteries (1-3).

The interstitial pregnancy can be diagnosed early in many cases using the trans-vaginal ultrasound (TVS), and $\beta$-hCG, but the diagnosis can be challenging in some cases (4). The missed diagnosis of interstitial ectopic pregnancy may result in life-threatening internal hemorrhage (4).

The criteria that may differentiate includes; empty uterus, with an eccentric gestational sac seen separate from the endometrium, the gestational sac is more than $1 \mathrm{~cm}$ away from the most lateral edge of the uterine cavity, and $<5$-mm myometrium surrounding the gestational sac (5). Moreover, an echogenic line (the interstitial line sign) extending from the gestational sac to the endometrium cavity represents the interstitial portion of the fallopian tube, and is highly sensitive, and specific (6). In unclear cases; the three-dimensional sonography may help the diagnosis (7). 
Traditionally interstitial pregnancy treated with open laparotomy, and salpingectomy with possible need for blood transfusion, and other treatment options include; laparoscopic salpingectomy, and systemic methotrexate for un-ruptured ectopic pregnancies in hemodynamically stable patients (8).

\section{Case Report}

29 years-old woman, Gravida 3, Para 2 (previous two vaginal deliveries), admitted to the hospital with severe abdominal pain, dizziness, and syncope, positive pregnancy test, and missed period for 3 days. She used to have regular menses, and her $\beta$-hCG done on admission was $3390 \mathrm{mIU} / \mathrm{mL}$.

She was pale on admission, she had tachycardia (pulse rate 120 beats/minute) with generalized abdominal pain, distension, and tenderness.

Trans-vaginal ultrasound (TVS) showed empty uterine cavity, free pelvic fluid in the Douglas pouch (DP) (Figure 1), with severe cervical motion tenderness. She was diagnosed as acutely disturbed ectopic pregnancy. Emergency laparotomy done revealed ruptured right interstitial pregnancy (Figures 2 and 3).

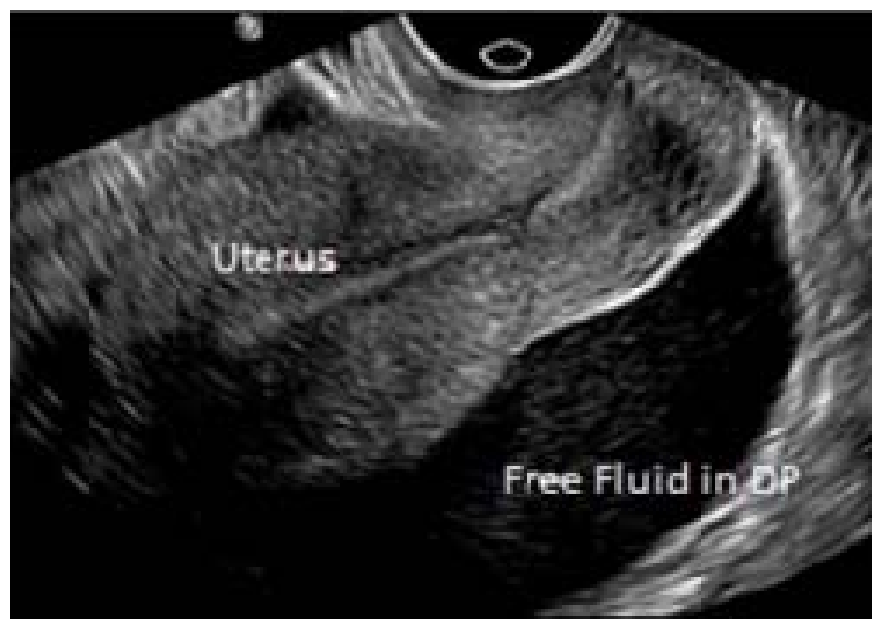

Figure 1: Trans-vaginal ultrasound image of empty uterus and free fluid in Douglas pouch

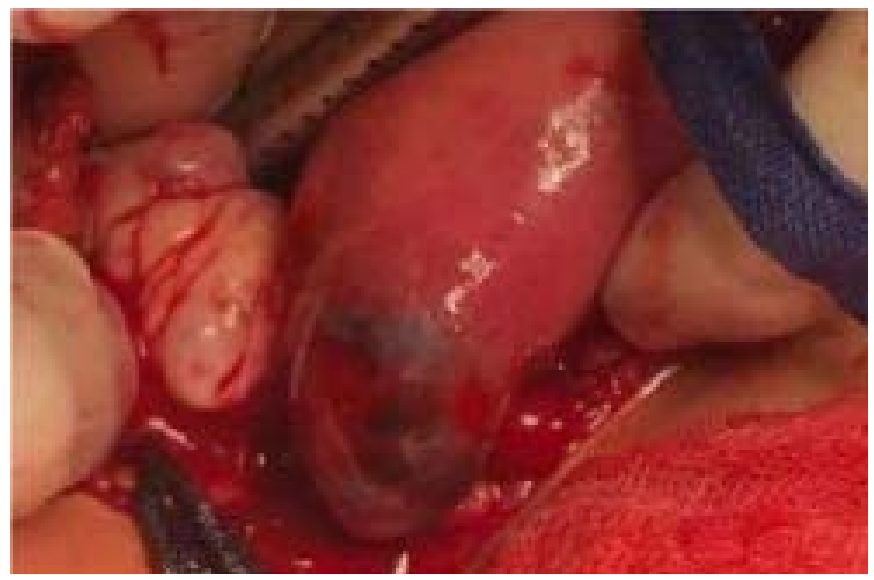

Figure 2: Intra-operative image of right interstitial ectopic pregnancy

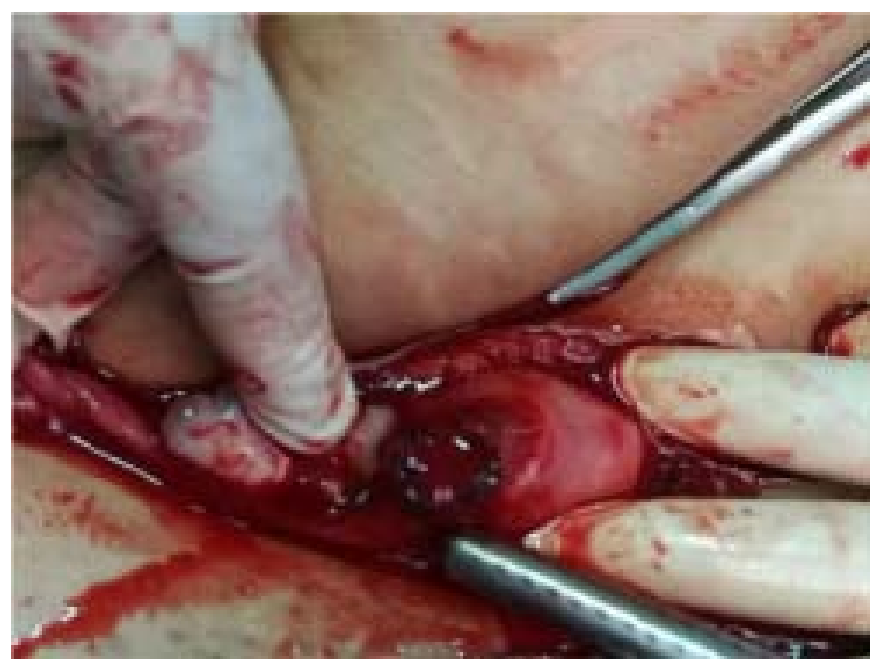

Figure 3: Intra-operative image of right interstitial ectopic pregnancy

The ruptured pregnancy managed by right salpingectomy, and hemostatic stitches at the right uterine corn.

Her hemoglobin was 8 gr on admission, and 3 units of packed red blood cells (one intra-operative, and two post-operative) were transfused. Her post-operative hemoglobin was $10.5 \mathrm{gr}$, and she was discharged from the hospital on the 3rd post-operative day in good general condition.

\section{Discussion}

Previous ectopic pregnancy, pelvic inflammatory diseases (PID), fallopian tube surgery or sterilization, documented tubal pathology, assisted reproductive techniques (ARTs), multiple sexual partners, prior cesarean delivery are risk factors of ectopic pregnancy (9).

Approximately $92 \%$ of ectopic pregnancies occur in the ampulla part of the fallopian tubes, and rupture of the ampulla ectopic pregnancy usually occurs at 8-12 weeks. While, 2.5\% of the ectopic pregnancies are interstitial ectopic pregnancies, and less commonly cervical, ovary and/or peritoneal (9).

Interstitial pregnancy can have disturbed 8-16 weeks of amenorrhea (later than the distal tubal ectopic pregnancy), with subsequent massive internal hemorrhage.

An ectopic pregnancy should be suspected if the TVS shows no intrauterine gestational sac, and $\beta$-hCG is higher than $1,500 \mathrm{mIU} / \mathrm{mL}(10-11)$.

In spite of the available diagnostic tools as TVS, and $\beta$ $\mathrm{hCG}$, the diagnosis of interstitial ectopic pregnancy can be challenging in some cases, and the missed diagnosis of interstitial ectopic pregnancy may result in life-threatening internal hemorrhage (4).

The obstetricians should be aware by the rare varieties of the ectopic pregnancies such as the interstitial pregnancy to 
avoid the massive internal hemorrhage, and subsequent significant morbidity.

Traditionally interstitial pregnancy treated with open laparotomy (8). A Cochrane systematic review concluded that the laparoscopic conservative surgery is significantly less successful than the open surgical approach in the elimination of tubal pregnancy due to the higher persistent trophoblast rate after laparoscopic surgery, and one single dose of methotrexate necessitating additional methotrexate injections or surgical interventions (12).

Katz et al, (13), reported two cases of interstitial pregnancy treated successfully with a combined hysteroscopic, and laparoscopic approach (13).

Timmerman et al, (14), reported successful treatment of interstitial ectopic pregnancy by systemic methotrexate (multiple dose regimen) in two patients out of three. In addition; Tanaka et al, (15), reported successful treatment of interstitial ectopic pregnancy in $93.9 \%$ (31/33) women with bolus dose of methotrexate $100 \mathrm{mg}$ followed by $200 \mathrm{mg}$ of methotrexate infusion over 12 hours, and 4 doses of $15 \mathrm{mg}$ oral folinic acid post-treatment.

Interstitial pregnancy is a rare form of ectopic pregnancy, the obstetricians should be aware of rare forms of ectopic pregnancies, it can have disturbed few days or weeks after the missed period leading to subsequent significant morbidity.

:Acknowledgements: None

Financial / Material Support: None

Conflict of Interest: The authors declare no conflict of interest.

\section{References}

1. Tulandi T, Al-Jaroudi D. Interstitial pregnancy: results generated from the Society of Reproductive Surgeons Registry. Obstet Gynecol 2004;103(1):47-50.

2. Chopra S, Keepanasseril A, Rohilla M, Bagga R, Kalra J, Jain V. Obstetric morbidity and the diagnostic dilemma in pregnancy in rudimentary horn: retrospective analysis. Arch Gynecol Obstet 2009;280(6):907-10.

3. Pluchino N, Ninni F, Angioni S, Carmignani A, Genazzani AR, Cela V. Spontaneous cornual pregnancy after homo- lateral salpingectomy for an earlier tubal pregnancy: a case report and literature review. J Minim Invasive Gynecol. 2009;16(2):208-11.

4. Walid MS, Heaton RL. Diagnosis and laparoscopic treatment of cornual ectopic pregnancy. Ger Med Sci 2010 Jul 27;8. pii: Doc16.

5. Timor-Tritsch IE, Monteagudo A, Matera C, Veit CR. Sonographic evolution of cornual pregnancies treated without surgery. Obstet Gynecol 1992;79(6):1044-9.

6. Ackerman TE, Levi CS, Dashefsky SM, Holt SC, Lindsay DJ. Interstitial line: sonographic finding in interstitial (cornual) ectopic pregnancy. Radiology 1993;189(1):83-7.

7. Parker RA 3rd, Yano M, Tai AW, Friedman M, Narra VR, Menias CO. MR imaging findings of ectopic pregnancy: a pictorial review. Radiographics 2012; 32(5):1445-60; discussion 1460-2.

8. Tenore JL. Ectopic pregnancy. Am Fam Physician. 2000; 61(4):1080-8.

9. Agrawal R, Kumar P. Ectopic pregnancy in tubal stump after ipsilateral salpigo-oohorectomy: An unusual and rare case report. Journal of Infertility and Reproductive Biology 2015;3(40):234-6.

10. Lozeau AM, Potter B. Diagnosis and management of ectopic pregnancy. Am Fam Physician. 2005; 72(9):170714.

11. Nama V and Manyonda I. Tubal ectopic pregnancy: diagnosis and management. Arch Gynecol Obstet 2009:279: 443 .

12. Hajenius PJ, Mol F, Mol BW, Bossuyt PM, Ankum WM, van der Veen F Interventions for tubal ectopic pregnancy. Cochrane Database Syst Rev 2007 Jan 24;(1):CD000324.

13. Katz DL, Barrett JP, Sanfilippo JS, Badway DM. Combined hysteroscopy and laparoscopy in the treatment of interstitial pregnancy. Am J Obstet Gynecol 2003; 188(4):1113-4.

14. Timmerman E, Roovers JP, Ankum WM, Hajenius PJ. [Interstitial pregnancy: a rare type of ectopic pregnancy]. Ned Tijdschr Geneeskd 2008;152(14):787-91.

15. Tanaka K, Baartz D, Khoo SK. Management of interstitial ectopic pregnancy with intravenous methotrexate: An extended study of a standardised regimen. Aust N Z J Obstet Gynaecol. 2015;55(2):176-80. 